
T. Langer
E. Carlesso
A. Protti
M. Monti
B. Comini
L. Zani
D. T. Andreis
G. E. Iapichino
D. Dondossola
P. Caironi
S. Gatti
L. Gattinoni

Received: 24 August 2011

Accepted: 24 November 2011

Published online: 25 January 2012

(C) Copyright jointly held by Springer and ESICM 2012

Electronic supplementary material

The online version of this article (doi:10.1007/s00134-011-2455-2) contains supplementary material, which is available to authorized users.

T. Langer - E. Carlesso - A. Protti ·

M. Monti · B. Comini · L. Zani .

D. T. Andreis - G. E. Iapichino •

P. Caironi · L. Gattinoni (®)

Dipartimento di Anestesiologia,

Terapia Intensiva e Scienze

Dermatologiche, Fondazione IRCCS Ca'

Granda Ospedale Maggiore Policlinico

di Milano, Università degli Studi,

Via Francesco Sforza, 35

20122 Milano, Italy

e-mail: gattinon@policlinico.mi.it

Tel.: +39-02-55033232

Fax: +39-02-55033230

D. Dondossola $\cdot$ S. Gatti

Centro di Ricerche Chirurgiche Precliniche,

Fondazione IRCCS Ca' Granda Ospedale

Maggiore Policlinico di Milano,

Milan, Italy

\title{
In vivo conditioning of acid-base equilibrium by crystalloid solutions: an experimental study on pigs
}

P. Caironi · L. Gattinoni Dipartimento di Anestesia,

Rianimazione (Intensiva e Subintensiva) e Terapia del Dolore, Fondazione IRCCS Ca' Granda Ospedale Maggiore Policlinico di, Milano, Italy

Abstract Purpose: Large infusion of crystalloids may induce acid-base alterations according to their strong ion difference ([SID]). We wanted to prove in vivo, at constant $\mathrm{PCO}_{2}$, that if the [SID] of the infused crystalloid is equal to baseline plasma bicarbonate, the arterial $\mathrm{pH}$ remains unchanged, if lower it decreases, and if higher it increases. Methods: In 12 pigs, anesthetized and mechanically ventilated at $\mathrm{PCO}_{2} \approx 40 \mathrm{mmHg}$, 2.21 of crystalloids with a [SID] similar to (lactated Ringer's $28.3 \mathrm{mEq} / \mathrm{l}$ ), lower than (normal saline $0 \mathrm{mEq} / \mathrm{l}$ ), and greater than (rehydrating III $55 \mathrm{mEq} / \mathrm{l}$ ) baseline bicarbonate $(29.22 \pm 2.72 \mathrm{mEq} / \mathrm{l})$ were infused for $120 \mathrm{~min}$ in randomized sequence. Four hours of wash-out were allowed between the infusions. Every $30 \mathrm{~min}$ up to minute 120 we measured blood gases, plasma electrolytes, urinary volume, $\mathrm{pH}$, and electrolytes. Albumin, hemoglobin, and phosphates were measured at time 0 and 120 min. Results: Lactated Ringer's maintained arterial $\mathrm{pH}$ unchanged (from $7.47 \pm 0.06$ to $7.47 \pm 0.03)$ despite a plasma dilution around $12 \%$. Normal saline caused a reduction in $\mathrm{pH}$ (from $7.49 \pm 0.03$ to $7.42 \pm 0.04$ ) and rehydrating III induced an increase in $\mathrm{pH}$ (from $7.46 \pm 0.05$ to $7.49 \pm 0.04)$. The kidney reacted to the infusion, minimizing the acidbase alterations, by increasing/ decreasing the urinary anion gap, primarily by changing sodium and chloride concentrations. Lower urine volume after normal saline infusion was possibly due to its greater osmolarity and chloride concentration as compared to the other solutions. Conclusions: Results support the hypothesis that at constant $\mathrm{PCO}_{2}, \mathrm{pH}$ changes are predictable from the difference between the [SID] of the infused solution and baseline plasma bicarbonate concentration.

Keywords Crystalloid solutions . Acid-base equilibrium .

Stewart's approach .

Volume resuscitation .

Animal study 


\section{Introduction}

Fluid resuscitation with large amounts of crystalloids is usual in critically ill patients, and normal saline is the most widely used solution for this purpose. It has been observed that large amounts of normal saline induce metabolic acidosis [1-3]. According to misinterpreted Stewart's model $[4,5]$, this metabolic acidosis is due to a reduction in the plasma strong ion difference ([SID]). However, to fully understand the relationship among the dilution, kind of solution, and acid-base equilibrium, all three independent variables of Stewart's model must be considered together, not only [SID], but also $\mathrm{PCO}_{2}$ and the total amount of weak acids [Атот] [6]. Normal $\mathrm{pH}$ is assured by an adequate proportion of these components, whose individual variation may have opposite effects on $\mathrm{pH}$. As an example, a decrease in $\mathrm{PCO}_{2}$ and Атот causes alkalosis, while a decrease in [SID] causes acidosis. Therefore, a correct application of Stewart's model provides the same results as the classical bicarbonate/base excess approach [6, 7]. However, we believe that Stewart's approach has the great advantage to fully integrate the electrolyte and the acid-base equilibrium.

Whatever crystalloid solution not containing weak anions, before the infusion, has $\mathrm{PCO}_{2}$, [Атот], and [SID] values equal to zero. Crystalloids containing organic ions, such as lactated Ringer's, develop a positive [SID] only after the associated organic ions (such as lactate and acetate) are metabolized $[8,9]$. Therefore, when a crystalloid solution is added to normal plasma $\left(\mathrm{PCO}_{2} 40\right.$ $\mathrm{mmHg}$, [Атот] $17 \mathrm{mmol} / \mathrm{l}$, SID $42 \mathrm{mEq} / \mathrm{l}, \mathrm{pH}$ 7.4) in a closed system, the independent variables are equally diluted, and $\mathrm{pH}$ remains constant-for example, a $100 \%$ dilution of normal plasma leads to $\mathrm{PCO}_{2}$ of nearly 20 $\mathrm{mmHg}$, Атот of $8.5 \mathrm{mmol} / \mathrm{l}$, [SID] of $21 \mathrm{mEq} / \mathrm{l}$, and $\mathrm{pH}$ of 7.4. When $\mathrm{PCO}_{2}$ increases from 20 to $40 \mathrm{mmHg}$, opening the closed system to gases, $\mathrm{pH}$ decreases, with [Атот] and [SID] being unchanged [8]. Of course, by manipulating also the actual [SID] of the infused solution, the $\mathrm{pH}$ will change accordingly.

Provided the same mechanisms operate in vivo, we may expect that a proper combination of $\mathrm{PCO}_{2}$, [SID], and Атот could manipulate the acid-base equilibrium toward acidosis, alkalosis, or unchanged $\mathrm{pH}$. Actually, it was shown that no acidosis developed in normal animals when the diluting solution had a [SID] of nearly $24 \mathrm{mEq} / 1$ [9]. We expanded this observation, showing in vitro that plasma $\mathrm{pH}$ after crystalloid dilution at constant $\mathrm{PCO}_{2}$ depends on the relationship between infused [SID] ([SID $]_{\text {inf }}$ ) and the baseline plasma bicarbonate concentration $\left(\left[\mathrm{HCO}_{3}^{-}\right]_{\text {baseline }}\right.$ ) (see the Online Data Supplement for the mathematical derivation). At constant $\mathrm{PCO}_{2}$, when $[\mathrm{SID}]_{\text {inf }}-\left[\mathrm{HCO}_{3}^{-}\right]_{\text {baseline }}=0$ no change in $\mathrm{pH}$ occurs, whatever the degree of dilution. When $[\mathrm{SID}]_{\text {inf }}-\left[\mathrm{HCO}_{3}^{-}\right]_{\text {baseline }}>0 \mathrm{pH}$ increases, while it decreases when $[\mathrm{SID}]_{\text {inf }}-\left[\mathrm{HCO}_{3}^{-}\right]_{\text {baseline }}<0[7]$.
As these rules were identified in vitro, we aimed to confirm them in vivo, considering the animals as a "black box." In these experiments, we describe the acid-base equilibrium of the black box for a given input (crystalloid infusion) and output (urine volume and electrolyte composition). If the rules identified in vitro apply in vivo, changes in the acid-base equilibrium following the infusion of whatever crystalloid solution in subjects undergoing volume replacement will be easily predictable also in clinical practice.

\section{Materials and methods}

The study was approved by the Institutional Review Board and conducted in accordance with international recommendations [10]. Twelve healthy female pigs weighing $22 \pm 3 \mathrm{~kg}$ were anesthetized and mechanically ventilated (Engstrom Carestation, GE Healthcare) with PEEP $5 \mathrm{~cm} \mathrm{H} \mathrm{H}_{2} \mathrm{O}$, tidal volume $10 \mathrm{ml} / \mathrm{kg}$ and respiratory rate adjusted to maintain $\mathrm{PCO}_{2}$ constant throughout the experiment by capnography. Arterial, central venous, and bladder catheters were surgically placed [11]. Two hours of stabilization preceded the beginning of the protocol.

\section{Study protocol}

In each pig, normal saline (Baxter spa, Roma, Italy), lactated Ringer's (Fresenius Kabi Italia spa, Verona, Italy), and rehydrating III (Monico spa, Venezia/Mestre, Italy) were infused (see Table 1 for compositions). Six different sequences of dilution were possible, so pigs were randomly assigned to a sequence of dilutions to assure a

Table 1 Characteristics of the crystalloid solutions

\begin{tabular}{lcll}
\hline & $\begin{array}{l}\text { Rehydrating } \\
\text { III (RIII) }\end{array}$ & $\begin{array}{l}\text { Lactated } \\
\text { Ringer's (LR) }\end{array}$ & $\begin{array}{l}\text { Normal saline } \\
(\mathrm{NS})\end{array}$ \\
\hline$\left[\mathrm{Na}^{+}\right](\mathrm{mEq} / \mathrm{l})$ & 140.0 & 130.9 & 154.0 \\
{$\left[\mathrm{Cl}^{-}\right](\mathrm{mEq} / \mathrm{l})$} & 103.0 & 111.7 & 154.0 \\
{$\left[\mathrm{~K}^{+}\right](\mathrm{mEq} / \mathrm{l})$} & 10.0 & 5.4 & 0.0 \\
{$\left[\mathrm{Ca}^{2+}\right](\mathrm{mEq} / \mathrm{l})$} & 5.0 & 3.7 & 0.0 \\
{$\left[\mathrm{Mg}^{2+}\right](\mathrm{mEq} / \mathrm{l})$} & 3.0 & 0.0 & 0.0 \\
{$\left[\mathrm{Lac}^{-}\right](\mathrm{mEq} / \mathrm{l})$} & 0.0 & 28.3 & 0.0 \\
{$\left[\mathrm{Acetate}^{-}\right](\mathrm{mEq} / \mathrm{l})$} & 47 & 0 & 0 \\
{$\left[\mathrm{Citrate}{ }^{-}\right](\mathrm{mEq} / \mathrm{l})$} & 8 & 0 & 0 \\
{$[\mathrm{SID}](\mathrm{mEq} / \mathrm{l})$} & 55.0 & 28.3 & 0.0 \\
Osmolarity (mOsm/l) & 307.0 & 278.0 & 308.0
\end{tabular}

All concentrations are expressed as $\mathrm{mEq} / \mathrm{l}$. Osmolarity is expressed as $\mathrm{mOsm} / \mathrm{l}$

$\mathrm{Na}^{+}$sodium concentration, $\mathrm{Cl}^{-}$chloride concentration, $\mathrm{K}^{+}$potassium concentration, $\mathrm{Ca}^{2+}$ calcium concentration, $\mathrm{Mg}^{2+}$ magnesium concentration, Lac $^{-}$lactate concentration, Acetate ${ }^{-}$acetate concentration, Citrate ${ }^{-}$citrate concentration, SID strong ion difference, Osmolarity declared osmolarity 
balanced distribution (see Fig. 1 in the Online Data Supplement for study design). Each experimental set consisted of the 2-h infusion of the assigned crystalloid solution, in the amount of $10 \%$ of baseline body weight, followed by a 4-h 'wash-out' period, with the same fluid being infused at a rate of $3 \mathrm{ml} / \mathrm{h}$ to keep the vein open. Thereafter, a new experimental set began or, in the case of the last study period, the animal was killed with the injection of a bolus of $40 \mathrm{mEq}$ of potassium.

\section{Data collection}

During the 2-h study period, data were collected at baseline and every $30 \mathrm{~min}$. Thereafter, during the wash-out period, data were recorded every $2 \mathrm{~h}$ (minute 240 and 360). For each point, data on arterial blood gases, electrolytes, and acid-base variables (ABL800, Radiometer $\mathrm{GmbH}$, Germany) were recorded. Urinary output measurement and analysis of urinary $\mathrm{pH}\left(\mathrm{pH}_{\mathrm{U}}\right)$ and urinary electrolytes (sodium $\left[\mathrm{Na}^{+}\right]_{\mathrm{U}}$, potassium $\left[\mathrm{K}^{+}\right]_{\mathrm{U}}$, chloride $\left[\mathrm{Cl}^{-}\right]_{\mathrm{U}}$, and ammonium $\left[\mathrm{NH}_{4}^{+}\right]_{\mathrm{U}}$ ) were performed by the urinary analyzer K.IN.G ${ }^{\circledR}$ (collaboration of Orvim and Kardia, Milan, Italy) [12] and synchronized with blood data collection. For each dilution, samples for measurement of hemoglobin, albumin, and phosphates concentrations were sent to the central laboratory at time $0,120,240$ and $360 \mathrm{~min}$.

\section{Definitions}

Fluid balance was computed as $\sum$ Infused fluid$\sum$ Urinary output. Hemoglobin-derived $\left(\mathrm{DF}_{\mathrm{Hb}}\right)$ and albumin-derived dilution $\left(\mathrm{DF}_{\mathrm{Alb}}\right)$ were computed as previously described [13] and used to estimate plasma dilution. The plasma [SID] and urinary anion gap $[\mathrm{AG}]_{\mathrm{U}}$ were computed using standard formulas. The concentration of dissociated weak acids $\left[\mathrm{A}^{-}\right]$was calculated from plasma albumin and phosphates concentration as previously described [14], and the corresponding [Атот] was computed rearranging the dissociation equation for weak acids. Effective plasma osmolarity was calculated with the standard formula from sodium and glucose (see the Online Data Supplement for further details and equations).

\section{Statistical analysis}

Data are presented as mean \pm standard deviation. Twoway ANOVA RM was used to test the effects of different crystalloids and time and Bonferroni's $t$ test for multiple comparison. A rank transformation was used in the case of variables that were not normally distributed and did not pass the equal variance test. Infusion and wash-out (plus baseline) were tested separately. Linear regression was used to assess the level of agreement between variables.
Table 2 Baseline plasma and urinary acid-base variables

\begin{tabular}{lr}
\hline $\mathrm{PCO}_{2}(\mathrm{mmHg})$ & $40.4 \pm 2.7$ \\
{$[\mathrm{SID}](\mathrm{mEq} / \mathrm{l})$} & $40.43 \pm 2.14$ \\
{$\left[\mathrm{~A}_{\mathrm{TOT}}\right](\mathrm{mmol} / \mathrm{l})$} & $13.75 \pm 1.73$ \\
{$[\mathrm{Alb}](\mathrm{g} / \mathrm{l})$} & $23.4 \pm 4$ \\
{$[\mathrm{Phosph}(\mathrm{mmol} / \mathrm{l})$} & $2.67 \pm 0.36$ \\
{$[\mathrm{BE}](\mathrm{mmol} / \mathrm{l})$} & $5.47 \pm 2.78$ \\
$\mathrm{pHa}$ & $7.47 \pm 0.04$ \\
{$\left[\mathrm{HCO}_{3}{ }^{-}\right](\mathrm{mmol} / \mathrm{l})$} & $29.22 \pm 2.72$ \\
{$\left[\mathrm{Na}^{+}\right](\mathrm{mEq} / \mathrm{l})$} & $138 \pm 2.73$ \\
{$\left[\mathrm{Cl}^{-}\right]_{(\mathrm{mEq} / \mathrm{l})}$} & $103.08 \pm 3.32$ \\
{$\left[\mathrm{~K}^{+}\right]_{(\mathrm{mEq} / \mathrm{l})}$} & $3.78 \pm 0.34$ \\
{$\left[\mathrm{Ca}^{2+}\right](\mathrm{mEq} / \mathrm{l})$} & $2.60 \pm 0.22$ \\
{$\left[\mathrm{Lac}^{-}\right](\mathrm{mEq} / \mathrm{l})$} & $0.88 \pm 0.25$ \\
$\mathrm{Osmolarity}(\mathrm{mOsm} / \mathrm{l})^{\mathrm{pH}_{\mathrm{U}}}$ & $281.1 \pm 5.1$ \\
{$\left[\mathrm{Na}^{+}\right]_{\mathrm{U}}(\mathrm{mEq} / \mathrm{l})$} & $6.49 \pm 0.6$ \\
{$\left[\mathrm{Cl}^{-}\right]_{\mathrm{U}}(\mathrm{mEq} / \mathrm{l})$} & $58.0 \pm 23.2$ \\
{$\left[\mathrm{~K}^{+}\right]_{\mathrm{U}}(\mathrm{mEq} / \mathrm{l})$} & $127.8 \pm 68.5$ \\
{$\left[\mathrm{NH}_{4}^{+}\right]_{\mathrm{U}}(\mathrm{mEq} / \mathrm{l})$} & $46.1 \pm 17.4$ \\
{$[\mathrm{AG}]_{\mathrm{U}}(\mathrm{mEq} / \mathrm{l})$} & $16.0 \pm 13.1$ \\
\hline
\end{tabular}

All values are expressed as mean \pm standard deviation $\mathrm{PCO}_{2}$ partial pressure of carbon dioxide, SID strong ion difference, Aтот plasma concentration of non-carbonic weak acids, $A l b$ albumin concentration, Phosph phosphate concentration, $B E$ base excess, $\mathrm{pHa}$ arterial $\mathrm{pH}, \mathrm{HCO}_{3}^{-}$bicarbonate concentration, $\mathrm{Na}^{+}$ sodium concentration, $\mathrm{Cl}^{-}$chloride concentration, $\mathrm{K}^{+}$potassium concentration, $\mathrm{Ca}^{2+}$ calcium concentration, $\mathrm{Lac}^{-}$lactate concentration, Osmolarity effective plasma osmolarity, $[\mathrm{pH}]_{\mathrm{U}}$ urinary $\mathrm{pH}$, $\left[\mathrm{Na}^{+}\right]_{U}$ urinary sodium concentration, $\left[\mathrm{Cl}^{-}\right]_{U}$ urinary chloride concentration, $\left[\mathrm{K}^{+}\right]_{\mathrm{U}}$ urinary potassium concentration, $\left[\mathrm{NH}_{4}^{+}\right]_{U}$ urinary ammonium concentration, $[A G]_{U}$ urinary anion gap

Paired $t$ test was used to compare $\mathrm{DF}_{\mathrm{Hb}}$ and $\mathrm{DF}_{\mathrm{Alb}}$. Statistical significance was defined as $p<0.05$. Analysis was performed with SAS v.9.2. (SAS, Cary, NC) and SigmaPlot v.11.2 (Systat Software Inc., San Jose, CA).

\section{Results}

Baseline characteristics

Baseline acid-base variables and electrolytes in plasma and urine are summarized in Table 2. Of note, before any intervention all pigs presented slight metabolic alkalosis and hypoalbuminemia. However, all values are consistent with previously reported data on acid-base variables in healthy piglets weighing between 20 and $25 \mathrm{~kg}$, which, compared to humans, present higher $\mathrm{pH}(7.48 \pm 0.038)$, similar $\mathrm{PCO}_{2}(40 \pm 2.8 \mathrm{mmHg})$, higher bicarbonate concentrations $(29 \pm 2.2 \mathrm{mEq} / \mathrm{l})$, and lower albumin concentrations $(25.4 \pm 2.08 \mathrm{~g} / \mathrm{l})$ [15].

\section{Effective dilution}

Table 3 summarizes the principal data concerning infused fluids, urinary output, water balance, and consequent 
Table 3 Dilution variables at time 120

\begin{tabular}{lccc}
\hline & Rehydrating III (RIII) & Lactated Ringer's (LR) & Normal saline (NS) \\
\hline Infused fluid (ml) & $2,213 \pm 329$ & $2,213 \pm 329$ & $2,213 \pm 329$ \\
Urinary output (ml) & $907 \pm 254$ & $754 \pm 190$ & $644 \pm 251^{*}$ \\
Fluid balance (ml) & $1,306 \pm 452$ & $1,459 \pm 333$ & $1,569 \pm 347^{*}$ \\
Hb-derived plasma dilution (\%) & $12.20 \pm 9.47$ & $15.53 \pm 6.10$ & $10.92 \pm 11.06$ \\
Albumin-derived plasma dilution (\%) & $11.00 \pm 7.67$ & $14.66 \pm 5.27$ & $12.61 \pm 9.49$ \\
\hline
\end{tabular}

The table summarizes dilution parameters at the end of the infusion (120 min). Fluid balance was defined as infused fluid minus urinary output. See the Online Data Supplement for the $\mathrm{Hb}$ - and albumin-derived dilution explanation

$* p<0.05$ compared to rehydrating III
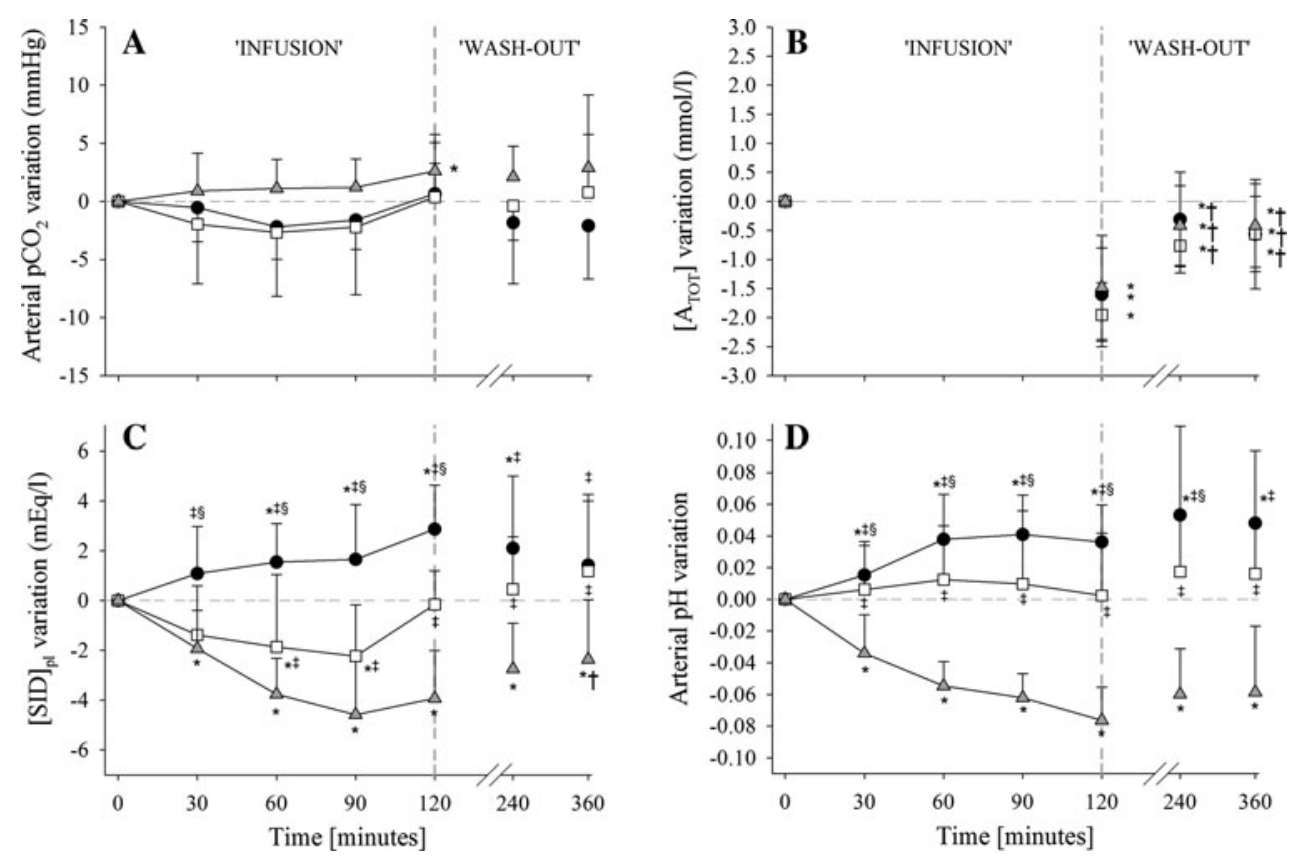

Fig. 1 Plasma variations in independent variables induced by the infusion of crystalloid solutions at different [SID]s $(\mathbf{a}, \mathbf{b}, \mathbf{c})$ and the consequent variations in plasma $\mathrm{pH}(\mathbf{d})$. Grey triangles represent variations induced by the infusion of normal saline. White squares represent variations induced by the infusion of lactated Ringer's solution. Black dots represent variations induced by the infusion of rehydrating III. All data are expressed as mean \pm standard deviation. [Атот] represents the plasma concentration of non-

carbonic weak acids; [АТот] was measured at baseline, at the end of the infusion, and at time 240 and $360 \mathrm{~min} ;[\mathrm{SID}]_{\mathrm{pl}}$ represents the plasma strong ion difference. $* p<0.05$ : Compared to baseline value of the same group. ${ }^{\dagger} p<0.05$ : Compared to time $120 \mathrm{~min}$ of the same group; ${ }^{*} p<0.05$ : Rehydrating III or lactated Ringer's compared to normal saline. ${ }^{\S} p<0.05$ Rehydrating III compared to lactated Ringer's. See text for additional explanations

effective plasma dilution at the end of the infusion period (time $120 \mathrm{~min}$ ), according to the infused crystalloid. The mean hemoglobin and albumin-derived plasma dilution coefficients were not significantly different $(12.9 \pm 9.1 \%$ and $12.8 \pm 7.6 \%$, respectively) and did not differ significantly among the crystalloids used. Of note, however, the urinary output was significantly lower and fluid balance more positive after the infusion of normal saline, compared to lactated Ringer's and rehydrating III infusion.

\section{Plasma variables}

Figure 1 shows the time course of the independent variables and the resulting $\mathrm{pH}$ as a function of the three crystalloid solutions. As shown in panel $\mathrm{A}, \mathrm{PCO}_{2}$ was maintained constant throughout the experiments, while [Атот] (panel B) was equally diluted by the three solutions. In contrast the time course of plasma [SID] variations (panel C) was significantly different when infusing solutions with different SIDs. These changes in 
Table 4 Variations in plasma acid-base variables, electrolytes, and osmolarity

\begin{tabular}{|c|c|c|c|c|c|c|c|}
\hline Time (min) & 0 & 30 & 60 & 90 & 120 & 240 & 360 \\
\hline \multicolumn{8}{|l|}{ Rehydrating III } \\
\hline $\begin{array}{l}\mathrm{d}\left[\mathrm{HCO}_{3}{ }^{-}\right](\mathrm{mmol} / \mathrm{l}) \\
\mathrm{P}_{\mathrm{TIME}} \\
\mathrm{P}_{\mathrm{INF}}\end{array}$ & $0.00 \pm 0.00$ & $\begin{array}{l}0.73 \pm 1.25 \\
\text { 林末 }\end{array}$ & $\begin{array}{l}1.19 \pm 1.20 \\
* * \\
\text { 林ฐ }\end{array}$ & $\begin{array}{l}1.75 \pm 1.67 \\
* * * \\
\text { 林ฐ§§ }\end{array}$ & $\begin{array}{l}3.23 \pm 1.71 \\
* * * \\
\text { t+* §§§ }\end{array}$ & $\begin{array}{l}2.51 \pm 2.43 \\
* * * \\
\text { 林§ }\end{array}$ & $\begin{array}{l}2.01 \pm 2.30 \\
* * * \dagger \\
\text { 林 }\end{array}$ \\
\hline $\begin{array}{l}\text { INF } \\
\text { dBE }(\mathrm{mmol} / \mathrm{l})\end{array}$ & $0.00 \pm 0.00$ & $0.84 \pm 0.99$ & $1.60 \pm 1.27$ & $2.13 \pm 1.64$ & $3.28 \pm 1.56$ & $2.94 \pm 2.52$ & $2.46 \pm 2.28$ \\
\hline $\begin{array}{l}\mathrm{P}_{\mathrm{TIME}} \\
\mathrm{P}_{\mathrm{INF}}\end{array}$ & & $\mathrm{t木 \textrm {A }}$ & $\begin{array}{l}* * * \\
\mathrm{t}+\mathrm{t} \S \S \S\end{array}$ & $\begin{array}{l}* * * \\
\text { 林‡§§§ }\end{array}$ & $\begin{array}{l}* * * \\
\text { 林§§§ }\end{array}$ & $\begin{array}{l}* * * \\
\text { 林§ }\end{array}$ & \\
\hline $\begin{array}{l}\mathrm{d}\left[\mathrm{Na}^{+}\right](\mathrm{mEq} / \mathrm{l}) \\
\mathrm{P}_{\mathrm{TIME}}\end{array}$ & $0.00 \pm 0.00$ & $0.67 \pm 1.07$ & $\underset{* *}{1.33} \pm 1.72$ & $\underset{* * *}{1.83} \pm 1.75$ & $\underset{* * *}{1.92} \pm 2.11$ & $0.83 \pm 2.59$ & $0.25 \pm 1.66$ \\
\hline $\mathrm{P}_{\mathrm{INF}}$ & & $\ddagger$ & 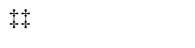 & 林 & 林 & $\star$ & 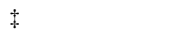 \\
\hline $\mathrm{d}\left[\mathrm{Cl}^{-}\right](\mathrm{mEq} / \mathrm{l})$ & $0.00 \pm 0.00$ & $-0.42 \pm 2.28$ & $-0.25 \pm 2.45$ & $0.08 \pm 2.78$ & $-1.08 \pm 2.61$ & $-1.58 \pm 4.44$ & $-1.50 \pm 3.12$ \\
\hline $\begin{array}{l}\mathrm{P}_{\mathrm{TIME}} \\
\mathrm{P}_{\mathrm{INF}}\end{array}$ & & $\mathrm{tt+}$ & 林 & 林 $\S$ & t⿰木木 & 林 & 林 \\
\hline $\begin{array}{l}\mathrm{d}\left[\mathrm{K}^{+}\right](\mathrm{mEq} / \mathrm{l}) \\
\mathrm{P}_{\mathrm{TIME}}\end{array}$ & $0.00 \pm 0.00$ & $0.04 \pm 0.14$ & $0.03 \pm 0.23$ & $-0.04 \pm 0.28$ & $-0.05 \pm 0.37$ & $-0.20 \pm 0.36$ & $-0.23 \pm 0.56$ \\
\hline $\mathrm{P}_{\mathrm{INF}}$ & & ttt & 林 & 林 & 林 & $\S \S$ & $\S \S$ \\
\hline $\mathrm{P}_{\mathrm{TIME}}\left[\right.$ Lactate $\left.^{-}\right](\mathrm{mEq} / \mathrm{l})$ & $0.00 \pm 0.00$ & $0.00 \pm 0.12$ & $0.03 \pm 0.16$ & $0.01 \pm 0.15$ & $0.07 \pm 0.12$ & $0.02 \pm 0.20$ & $-0.05 \pm 0.21$ \\
\hline $\mathrm{P}_{\mathrm{INF}}$ & & 林§§§ & 林 §§§ & 林§§§ & 林 $\S \S$ & 林 & \\
\hline $\mathrm{dOsm}(\mathrm{mOsm} / \mathrm{l})$ & $0.00 \pm 0.00$ & $1.19 \pm 1.99$ & $\begin{array}{l}2.49 \pm 3.12 \\
* * *\end{array}$ & $\underset{* * *}{3.43} \pm 3.14$ & $\begin{array}{l}3.65 \pm 3.91 \\
* * *\end{array}$ & $1.38 \pm 4.71$ & $\underset{+\uparrow \dagger}{0.34} \pm 3.06$ \\
\hline $\begin{array}{l}\text { TIME } \\
\mathrm{P}_{\mathrm{INF}}\end{array}$ & & t & 林 & 林 & 柿 & 林 & $\ddagger$ \\
\hline \multicolumn{8}{|l|}{ Lactated Ringer's } \\
\hline$\underset{\mathrm{P}_{\mathrm{TIME}}}{\mathrm{d}\left[\mathrm{HCO}_{3}{ }^{-}\right](\mathrm{mmol} / \mathrm{l})}$ & $0.00 \pm 0.00$ & $-0.86 \pm 1.51$ & $-0.83 \pm 2.00$ & $-0.69 \pm 1.01$ & $0.64 \pm 1.11$ & $1.07 \pm 1.35$ & ${\underset{* *}{* *}}^{1.52} \pm 2.54$ \\
\hline $\mathrm{P}_{\mathrm{INF}}$ & & & 林 & 林 & tht & 林 & 林 \\
\hline dBE (mmol/l) & $0.00 \pm 0.00$ & $-0.60 \pm 1.10$ & $-0.60 \pm 1.85$ & $-0.41 \pm 1.04$ & $0.62 \pm 1.16$ & $\underset{*}{1.14} \pm 1.55$ & $\begin{array}{l}1.44 \\
* *\end{array}$ \\
\hline $\begin{array}{l}\text { TIME } \\
\mathrm{P}_{\text {INF. }}\end{array}$ & & 杖 & 林 & 林 & 柿 & 林 & 郝 \\
\hline $\mathrm{d}\left[\mathrm{Na}^{+}\right](\mathrm{mEq} / \mathrm{l})$ & $0.00 \pm 0.00$ & $0.33 \pm 1.16$ & $\begin{array}{l}1.00 \pm 2.09 \\
*\end{array}$ & $\underset{*}{1.17} \pm 1.80$ & $\begin{array}{l}1.08 \pm 1.78 \\
*\end{array}$ & $0.00 \pm 2.30$ & $-0.82 \pm 2.36$ \\
\hline $\mathrm{P}_{\mathrm{INF}}$ & & tt & 林 & $\mathrm{at+}$ & 林 & 林 & 林 \\
\hline $\begin{array}{l}\mathrm{d}\left[\mathrm{Cl}^{-}\right](\mathrm{mEq} / \mathrm{l}) \\
\mathrm{P}_{\mathrm{TIME}}\end{array}$ & $0.00 \pm 0.00$ & $0.92 \pm 2.47$ & $\begin{array}{l}2.00 \pm 4.22 \\
*\end{array}$ & $\begin{array}{l}2.50 \\
* * *\end{array}$ & $0.67 \pm 2.35$ & $-0.17 \pm 2.59$ & $-1.73 \pm 3.80$ \\
\hline $\mathrm{P}_{\mathrm{INF}}$ & & 木 & 林 & $\mathrm{at+}$ & 林 & 林 & 林 \\
\hline $\begin{array}{l}\mathrm{d}\left[\mathrm{K}^{+}\right](\mathrm{mEq} / \mathrm{l}) \\
\mathrm{P}_{\mathrm{TIME}}\end{array}$ & $0.00 \pm 0.00$ & $-0.12 \pm 0.27$ & $-0.13 \pm 0.42$ & $-0.11 \pm 0.32$ & $0.04 \pm 0.25$ & $\begin{array}{l}0.25 \pm 0.27 \\
*\end{array}$ & $0.27 \pm 0.41$ \\
\hline $\mathrm{P}_{\mathrm{INF}}$ & & $\ddagger$ & 林 & 林 & ti & $\ddagger$ & \\
\hline $\begin{array}{l}\mathrm{d}\left[\text { Lactate }^{-}\right](\mathrm{mEq} / \mathrm{l}) \\
\mathrm{P}_{\text {TIME }} \\
\mathrm{P}_{\text {INF }}\end{array}$ & $0.00 \pm 0.00$ & $\begin{array}{l}0.68 \pm 0.22 \\
* * * \\
\text { 林 }\end{array}$ & $\begin{array}{l}0.74 \pm 0.22 \\
* * * \\
\text { 林 }\end{array}$ & $\begin{array}{l}0.79 \pm 0.31 \\
* * * \\
\mathrm{t+1}\end{array}$ & $\begin{array}{l}0.68 \pm 0.41 \\
* * * \\
t * t\end{array}$ & $-0.08 \pm 0.11$ & $-0.06 \pm 0.13$ \\
\hline $\begin{array}{l}\mathrm{dOsm}(\mathrm{mOsm} / \mathrm{l}) \\
\mathrm{P}_{\mathrm{TIME}}\end{array}$ & $0.00 \pm 0.00$ & $0.35 \pm 2.17$ & $\underset{* *}{1.71} \pm 3.71$ & $\underset{* * *}{2.20} \pm 3.45$ & $\begin{array}{l}2.35 \\
* * *\end{array}$ & $-0.04 \pm 4.52$ & $-1.86 \pm 4.58$ \\
\hline $\mathrm{P}_{\mathrm{INF}}$ & & 林 & 林 & 林 & 林 & 林 & 林 \\
\hline \multicolumn{8}{|l|}{ Normal saline } \\
\hline $\begin{array}{l}\mathrm{d}\left[\mathrm{HCO}_{3}{ }^{-}\right](\mathrm{mmol} / \mathrm{l}) \\
\mathrm{P}_{\mathrm{TIME}} \\
\mathrm{P}_{\mathrm{INF}}\end{array}$ & $0.00 \pm 0.00$ & $-1.83 \pm 1.20$ & $\begin{array}{l}-3.06 \pm 1.20 \\
* * *\end{array}$ & $\begin{array}{l}-3.44 \pm 1.60 \\
* * *\end{array}$ & $\begin{array}{l}-3.49 \pm 1.61 \\
* * *\end{array}$ & $\begin{array}{l}-2.68 \pm 1.29 \\
* * *\end{array}$ & $\underset{* * * \dagger}{-2.25} \pm 1.83$ \\
\hline $\begin{array}{l}\mathrm{dBE}(\mathrm{mmol} / \mathrm{l}) \\
\mathrm{P}_{\mathrm{TIME}} \\
\mathrm{P}_{\mathrm{INF}}\end{array}$ & $0.00 \pm 0.00$ & $-2.09 \pm 0.93$ & $\begin{array}{l}-3.48 \pm 0.99 \\
* * *\end{array}$ & $\begin{array}{l}-3.93 \pm 1.36 \\
* * *\end{array}$ & $\begin{array}{l}-4.20 \pm 1.30 \\
* * *\end{array}$ & $\begin{array}{l}-3.19 \pm 1.29 \\
* * *\end{array}$ & $\begin{array}{l}-2.88 \\
* * * \dagger\end{array}$ \\
\hline 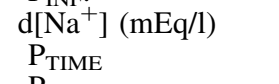 & $0.00 \pm 0.00$ & $\underset{* * *}{2.17} \pm 0.84$ & $\begin{array}{l}3.25 \pm 1.06 \\
* * *\end{array}$ & $\begin{array}{l}4.17 \pm 1.59 \\
* * *\end{array}$ & $\begin{array}{l}4.33 \pm 1.92 \\
* * *\end{array}$ & $\underset{* * *}{2.92} \pm 1.24$ & $\underset{* * *}{2.08} \pm 2.19$ \\
\hline $\begin{array}{l}\mathrm{P}_{\text {INF }} \\
\mathrm{d}\left[\mathrm{Cl}^{-}\right](\mathrm{mEq} / \mathrm{l}) \\
\mathrm{P}_{\mathrm{TIME}} \\
\mathrm{P}_{\mathrm{INF}}\end{array}$ & $0.00 \pm 0.00$ & $\begin{array}{l}3.75 \pm 1.77 \\
* * *\end{array}$ & $\begin{array}{l}6.67 \pm 1.37 \\
* * *\end{array}$ & $\begin{array}{l}8.50 \pm 3.12 \\
* * *\end{array}$ & $\begin{array}{l}8.08 \pm 2.61 \\
* * *\end{array}$ & $\begin{array}{l}5.75 \pm 2.22 \\
* * * \dagger \dagger\end{array}$ & $\begin{array}{l}4.58 \pm 3.18 \\
* * *+\dagger \dagger\end{array}$ \\
\hline $\begin{array}{l}\mathrm{d}\left[\mathrm{K}^{+}\right](\mathrm{mEq} / \mathrm{l}) \\
\mathrm{P}_{\mathrm{TIME}} \\
\mathrm{P}_{\mathrm{INF}}\end{array}$ & $0.00 \pm 0.00$ & $\begin{array}{l}-0.43 \pm 0.13 \\
* * *\end{array}$ & $\begin{array}{l}-0.54 \pm 0.17 \\
* * *\end{array}$ & $\begin{array}{l}-0.48 \pm 0.19 \\
* * *\end{array}$ & $\begin{array}{l}-0.45 \pm 0.34 \\
* * *\end{array}$ & $\underset{\dagger \dagger}{-0.13 \pm 0.30}$ & $-0.02 \pm 0.28$ \\
\hline
\end{tabular}


Table 4 continued

\begin{tabular}{|c|c|c|c|c|c|c|c|}
\hline Time (min) & 0 & 30 & 60 & 90 & 120 & 240 & 360 \\
\hline $\begin{array}{l}\mathrm{d}\left[\text { Lactate }^{-}\right](\mathrm{mEq} / \mathrm{l}) \\
\mathrm{P}_{\mathrm{TIME}} \\
\mathrm{P}_{\mathrm{INF}}\end{array}$ & $0.00 \pm 0.00$ & $\begin{array}{l}-0.12 \pm 0.07 \\
* * *\end{array}$ & $\begin{array}{l}-0.19 \pm 0.08 \\
* * *\end{array}$ & $\begin{array}{l}-0.20 \pm 0.13 \\
* * *\end{array}$ & $\begin{array}{l}-0.20 \pm 0.14 \\
* * *\end{array}$ & $\begin{array}{l}-0.17 \pm 0.14 \\
* * *\end{array}$ & $\begin{array}{l}-0.14 \pm 0.22 \\
* * *\end{array}$ \\
\hline $\begin{array}{l}\mathrm{dOsm}(\mathrm{mOsm} / \mathrm{l}) \\
\mathrm{P}_{\mathrm{TIME}} \\
\mathrm{P}_{\mathrm{INF}}\end{array}$ & $0.00 \pm 0.00$ & $\begin{array}{l}3.81 \pm 1.51 \\
* * *\end{array}$ & $\begin{array}{l}6.10 \pm 1.81 \\
* * *\end{array}$ & $\begin{array}{l}7.81 \pm 2.73 \\
* * *\end{array}$ & $\begin{array}{l}8.29 \pm 3.54 \\
* * *\end{array}$ & $\begin{array}{l}5.48 \pm 2.54 \\
* * *\end{array}$ & $\begin{array}{l}3.69 \pm 4.60 \\
* * *+\dagger\end{array}$ \\
\hline
\end{tabular}

The table summarizes variations in plasma acid-base variables, electrolytes, and osmolarity induced by the infusion of the crystalloid solutions. All data are expressed as mean \pm standard deviation

$d\left[\mathrm{HCO}_{3}{ }^{-}\right]$variations in bicarbonate concentration, $d \mathrm{BE}$ variations in base excess, $d\left[\mathrm{Na}^{+}\right]$variations in sodium concentration, $d\left[\mathrm{Cl}^{-}\right]$variations in chloride concentration, $d\left[K^{+}\right]$variations in potassium concentration, $d\left[\right.$ Lactate $\left.^{-}\right]$variations in lactate concentration, $d O s m$ variations in effective osmolarity

independent variables ([SID] and [Атот]) induced the variations in $\mathrm{pH}$ represented in panel $\mathrm{D}$. As shown, while the infusion of normal saline and rehydrating III caused significant variations in $\mathrm{pH}$ (reduction and increase, respectively), no variations were observed during the infusion of lactated Ringer's solution. Of note, the difference between baseline $\left[\mathrm{HCO}_{3}{ }^{-}\right]$and $[\mathrm{SID}]_{\text {inf }}$ expressed in $\mathrm{mEq} / \mathrm{l}$ was $+26.4 \pm 2.5$ for rehydrating III, $-29 \pm 2.7$ for normal saline, and $+1.05 \pm 2.35$ for lactated Ringer's solution.

\section{Plasma electrolytes and osmolarity}

The time course of the individual electrolytes as well as of osmolarity variations is reported in Table 4. As shown, compared to normal saline, rehydrating III and lactated Ringer's differed in the time course of $\left[\mathrm{Na}^{+}\right]$(lower increase), $\left[\mathrm{Cl}^{-}\right]$(lower increase), $\left[\mathrm{K}^{+}\right]$(significantly

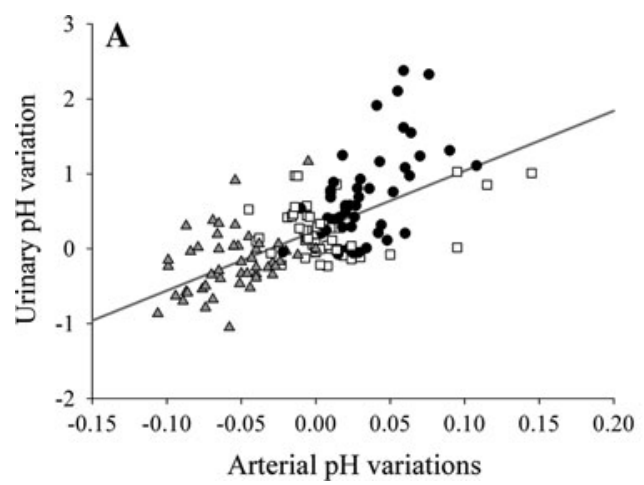

Fig. 2 Figure represents, at each experimental point during the infusion, the relationship between the urinary $\mathrm{pH}$ variations and the arterial $\mathrm{pH}$ variations (panel $A$, intercept $=0.24$, slope $=7.99$, $r^{2}=0.39, p<0.0001$ ), and the relationship between urinary $\mathrm{pH}$ variations and the urinary anion gap (panel $B$, intercept $=0.11$, slope $\left.=9.29 e-3, r^{2}=0.41, p<0.0001\right)$ during the infusion of
$* p<0.05$ versus baseline, $* * p<0.01$ versus baseline, $* * * p<0.001$ versus baseline, $\dagger p<0.05$ versus time 120 , †† $p<0.01$ versus time 120 , $\dagger \dagger p<0.001$ versus time 120 , $\$ p<0.05$ versus normal saline, ${ }^{\ddagger} p<0.01$ versus normal saline, th $p<0.001$ versus normal saline, ${ }^{\S} p<0.05$ versus lactated Ringer's, ${ }^{\S \S} p<0.01$ versus lactated Ringer's, ${ }^{\S \S \S} p<0.001$ versus lactated Ringer's. $\mathrm{P}_{\text {TIME }}$ defines statistics over time, i.e., compared to baseline value or to time 120 . $\mathrm{P}_{\mathrm{INF}}$ defines statistics among different types of crystalloids

decreased in normal saline), and lactate. Of note, the increase in effective osmolarity was remarkably greater with normal saline compared to the other two solutions.

\section{Urinary output}

As shown in Table 3, urinary output at the end of dilution significantly differed among the solutions, being significantly lower with normal saline. However, after the washout period $(4 \mathrm{~h})$, the urinary volume was similar among the three groups $(1,366 \pm 288 \mathrm{ml}$ for rehydrating III, $1,336 \pm 316 \mathrm{ml}$ for lactated Ringer's, and 1,263 \pm $265 \mathrm{ml}$ for normal saline).

In Fig. 2a we report the urinary $\mathrm{pH}$ changes as a function of arterial $\mathrm{pH}$ changes recorded at each infusion time and for each crystalloid solution. As shown, the urinary $\mathrm{pH}$ variations roughly paralleled the arterial $\mathrm{pH}$ variations, suggesting that the kidney reacted to the

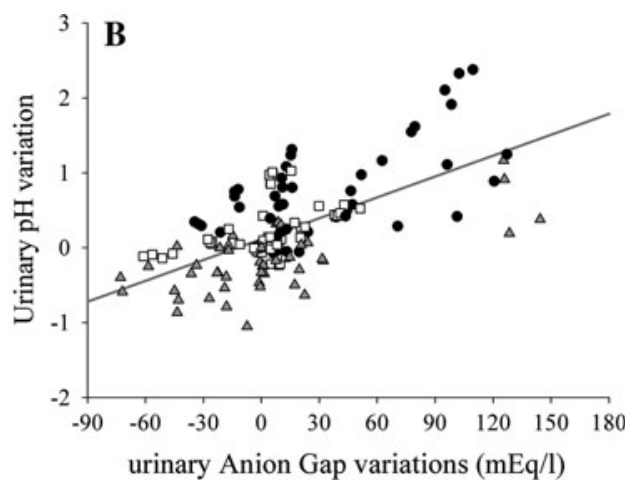

different crystalloid solutions. Grey triangles represent variations induced by the infusion of normal saline. White squares represent variations induced by the infusion of lactated Ringer's. Black dots represent variations induced by the infusion of rehydrating III. Grey solid lines represent linear regressions. See text for additional explanations 
acid-base plasma perturbations by trying to minimize them. This was likely obtained by readjusting the urinary [SID], one of the determinants of urinary $\mathrm{pH}$. Actually, as shown in Fig. $2 b$, the changes in the urinary anion gap $\left(\left[\mathrm{Na}^{+}\right]_{\mathrm{U}}+\left[\mathrm{K}^{+}\right]_{\mathrm{U}}-\left[\mathrm{Cl}^{-}\right]_{\mathrm{U}}\right)$, a surrogate for urinary [SID], were more negative during normal saline infusion, unchanged during lactated Ringer's infusion, and increased during rehydrating III infusion.

Pooling together all the experimental points, we found that the small variations in plasma [SID] were weakly but significantly correlated with the larger variations of the urinary anion gap $\left(r^{2}=0.07, p=0.0002\right)$, which in turn were significantly associated both with $\left[\mathrm{Na}^{+}\right]_{\mathrm{U}}\left(r^{2}=0.22\right.$, $p<0.0001)$ and $\left[\mathrm{Cl}^{-}\right]_{\mathrm{U}}\left(r^{2}=0.17, p<0.0001\right)$ changes (see Figures 2, 3, and 4 in the Online Data Supplement). In contrast, $\left[\mathrm{K}^{+}\right]_{\mathrm{U}}$ changes were unrelated to urinary anion gap changes, but were directly related to the potassium loaded during the infusions (see Figures 5, 6, 7 and Table 1 in the Online Data Supplement).

\section{Discussion}

The findings in this study are consistent with the hypothesis that in intact healthy animals at constant $\mathrm{PCO}_{2}$ the plasma $\mathrm{pH}$ can be predictably increased, decreased, or maintained unchanged, according to the difference between the baseline plasma $\left[\mathrm{HCO}_{3}{ }^{-}\right]$and the strong ion difference of the crystalloid solution infused. The theoretical background has been published previously [7] and is summarized in the Online Data Supplement. In vitro experiments with human plasma are consistent with the rules identified in theory, and, in the present study, we found that an intact animal behaves as the simple plasma model.

In fact, in the current work, three different solutions with $[\text { SID }]_{\text {inf }}$ greater than, similar to, or lower than the baseline plasma $\left[\mathrm{HCO}_{3}{ }^{-}\right]$were infused in each pig. Actually, the baseline $\left[\mathrm{HCO}_{3}^{-}\right]$was around $29 \mathrm{mEq} / 1$. When diluting with lactated Ringer's whose [SID] was similar to the baseline $\left[\mathrm{HCO}_{3}{ }^{-}\right]\left([\mathrm{SID}]_{\mathrm{inf}}=28.3 \mathrm{mEq} / \mathrm{l}\right.$ and $\left.\left[\mathrm{HCO}_{3}{ }^{-}\right]=29.35 \pm 2.35 \mathrm{mmol} / \mathrm{l}\right)$, no significant changes in $\mathrm{pH}$ were observed. In contrast, dilution with the other two solutions having [SID] $]_{\text {inf }}$ greater (rehydrating III, $[\mathrm{SID}]_{\mathrm{inf}}=55 \mathrm{mEq} / \mathrm{l}$ ) or smaller (normal saline, $\left.\mathrm{SID}_{\mathrm{inf}}=0 \mathrm{mEq} / \mathrm{l}\right)$ than baseline $\left[\mathrm{HCO}_{3}{ }^{-}\right]$determined a significant increase and reduction in $\mathrm{pH}$, respectively, as observed in vitro.

The plasma dilution we achieved, according to the reduction of the albumin and hemoglobin concentration averaged $12 \%$, a value that is consistent with the amount of fluid retained during the infusion period. In this study we did not measure or estimate the dilution of the different compartments, such as the interstititium and cellular space. However, the fact that the recorded changes in plasma $\mathrm{pH}$ were consistent with the ones observed during the dilution of isolated plasma suggests that also in vivo (regardless of the movements among the intravascular, interstitial, and intracellular space), the general rule found in vitro may be true, i.e., plasma changes during crystalloid infusion depend upon the difference between infused [SID] and baseline $\left[\mathrm{HCO}_{3}{ }^{-}\right]$. Our findings imply therefore that the body can be considered as a "black box" where, whatever the exchanges among plasma, interstitial fluid and cells are, the acid-base status can be easily predicted for any given input and output. While input conditions are easily computed (the kind of solution used and rate of delivery), the output has to be calculated from the urine volume and its electrolyte composition, at least of the electrolytes that are more relevant in determining the plasma [SID].

In this study we found that the response of the kidney to a given crystalloid solution is, in general, to preserve homeostasis. As far as the acid-base equilibrium is concerned, the kidney reacts by decreasing the urinary [SID], as inferred from the urinary anion gap, when infusing a solution causing acidosis and increasing it when infusing a solution causing alkalosis. This is a normal kidney "compensatory" response to plasma acid-base alterations $[16,17]$.

However, beyond the [SID] differences, the infused solution presented several other differences that could be relevant in determining urine volume and its composition, such as differences in osmolarity, potassium, and chloride load. While the volume receptors were likely equally stimulated by an identical volume load, the osmo-receptors were probably differently activated by the three solutions. In fact, after metabolism of the organic ions, lactated Ringer's and rehydrating III had an osmolarity that was about $50 \mathrm{mOsm} / \mathrm{l}$ lower than that of normal saline. The fact that lactate was nearly fully metabolized during lactated Ringer's infusion was documented by plasma lactate variations lower than $1 \mathrm{mmol} / \mathrm{l}$ (see Table 4). Therefore, the lower osmolarity of lactated Ringer's and rehydrating III via ADH suppression could have contributed to the greater urine volume caused by these infusions compared to the infusion of normal saline. Another possible factor influencing the urine volume and the electrolyte composition is the potassium load. Hypokalemia can cause a downregulation of aldosterone, with potassium-sparing effects and an increase of sodium plus water in the urine, whereas hyperkalemia can produce the opposite effects. Actually, in our experiments, we found that during normal saline infusion the plasma level of potassium was significantly reduced as well as its urinary concentration. In contrast, the infusion of lactated Ringer's and rehydrating III was associated with a significant increase in potassium excretion in proportion to the potassium load. Finally, the higher chloride concentration present in normal saline, per se, may have led to 
variations in glomerular filtration rates/renal arteriolar diameters $[18,19]$ with a consequent decrease in urinary volume. In summary, different mechanisms are likely to operate (sometimes in opposite directions) during the infusion, and all of them may have contributed to the urine pattern we recorded in these experiments.

We conclude that in healthy pigs with normal kidney function, the acid-base response to the infusion of solutions with different [SID]s is highly predictable and that the kidney response is specific for each solution, acting to restore the baseline conditions. If these findings could be applied to human beings, the response to large volume replacement of modifying plasma $\mathrm{pH}$ could be predicted. Although these experiments were conducted in healthy animals, the physicochemical basis of $\mathrm{pH}$ changes during crystalloid infusion should be the same in patients. It is however reasonable that the effects of crystalloid infusions on acid-base concentrations may be amplified in some conditions that are typical for critical illness, e.g., hypovolemia and renal failure [20-22]. Indeed, the physiological triggers for water and sodium elimination are not immediately activated in the first case, and in the case of renal failure, the kidney response to volume and electrolyte load is inadequate for the maintenance of homeostasis.

The call for a reasoned and rational use of crystalloid solutions is therefore straightforward, especially for critically ill patients.

Acknowledgments We are grateful to Fabio Ambrosetti for his technical support, to GE Healthcare for providing mechanical ventilators, to A. De Mori S.p.A., Italy, who kindly provided the blood gas analyzer, and to Roberto Castenetto for technical support with the KING analyzer. This study was supported in part by an Italian grant provided by Fondazione Fiera di Milano for Translational and Competitive Research (2007, Luciano Gattinoni).

\section{References}

1. Williams EL, Hildebrand KL, McCormick SA, Bedel MJ (1999) The effect of intravenous lactated Ringer's solution versus $0.9 \%$ sodium chloride solution on serum osmolality in human volunteers. Anesth Analg 88:999-1003

2. Scheingraber S, Rehm M, Sehmisch C, Finsterer U (1999) Rapid saline infusion produces hyperchloremic acidosis in patients undergoing gynecologic surgery. Anesthesiology 90:1265-1270

3. O'Malley CM, Frumento RJ, Hardy MA, Benvenisty AI, Brentjens TE, Mercer JS, Nett-Guerrero E (2005) A randomized, double-blind comparison of lactated Ringer's solution and $0.9 \%$ $\mathrm{NaCl}$ during renal transplantation. Anesth Analg 100:1518-1524, table

4. Fencl V, Leith DE (1993) Stewart's quantitative acid-base chemistry: applications in biology and medicine. Respir Physiol 91:1-16

5. Stewart PA (1983) Modern quantitative acid-base chemistry. Can J Physiol Pharmacol 61:1444-1461

6. Doberer D, Funk GC, Kirchner K, Schneeweiss B (2009) A critique of Stewart's approach: the chemical mechanism of dilutional acidosis. Intensive Care Med 35:2173-2180

7. Carlesso E, Maiocchi G, Tallarini F, Polli F, Valenza F, Cadringher P, Gattinoni L (2010) The rule regulating $\mathrm{pH}$ changes during crystalloid infusion. Intensive Care Med 37:461-468

8. Gattinoni L, Carlesso E, Maiocchi G, Polli F, Cadringher P (2009) Dilutional acidosis: where do the protons come from? Intensive Care Med 35:2033-2043
9. Morgan TJ, Venkatesh B, Hall J (2004) Crystalloid strong ion difference determines metabolic acid-base change during acute normovolaemic haemodilution. Intensive Care Med 30:1432-1437

10. Institute of Laboratory Animal Resources ColSNRC (1996) Guide for the care and use of laboratory animals. National Academy Press, Washington

11. Protti A, Cressoni M, Santini A, Langer T, Mietto C, Febres D, Chierichetti M, Coppola S, Conte G, Gatti S, Leopardi O, Masson S, Lombardi L, Lazzerini M, Rampoldi E, Cadringher P, Gattinoni L (2011) Lung stress and strain during mechanical ventilation: any safe threshold? Am J Respir Crit Care Med 183:1354-1362

12. Caironi $P$, Langer $T$, Taccone $P$, Bruzzone P, De CS, Vagginelli F, Caspani L, Marenghi C, Gattinoni L (2010) Kidney instant monitoring (K.IN.G): a new analyzer to monitor kidney function. Minerva Anestesiol 76:316-324

13. Ewaldsson CA, Hahn RG (2005) Kinetics and extravascular retention of acetated ringer's solution during isoflurane or propofol anesthesia for thyroid surgery. Anesthesiology 103:460-469

14. Fencl V, Jabor A, Kazda A, Figge J (2000) Diagnosis of metabolic acidbase disturbances in critically ill patients. Am J Respir Crit Care Med 162:2246-2251

15. Hannon JP, Bossone CA, Wade CE (1990) Normal physiological values for conscious pigs used in biomedical research. Lab Anim Sci 40:293-298
16. Gennari FJ, Goldstein MB, Schwartz WB (1972) The nature of the renal adaptation to chronic hypocapnia. J Clin Invest 51:1722-1730

17. Polak A, Haynie GD, Hays RM, Schwartz WB (1961) Effects of chronic hypercapnia on electrolyte and acidbase equilibrium. I. Adaptation. J Clin Invest 40:1223-1237

18. Hansen PB, Jensen BL, Skott O (1998) Chloride regulates afferent arteriolar contraction in response to depolarization. Hypertension 32:1066-1070

19. Wilcox CS (1983) Regulation of renal blood flow by plasma chloride. J Clin Invest 71:726-735

20. Bagshaw SM, George C, Dinu I, Bellomo R (2008) A multi-centre evaluation of the RIFLE criteria for early acute kidney injury in critically ill patients. Nephrol Dial Transplant 23:1203-1210

21. Bagshaw SM, Lapinsky S, Dial S, Arabi Y, Dodek P, Wood G, Ellis P, Guzman J, Marshall J, Parrillo JE, Skrobik Y, Kumar A (2009) Acute kidney injury in septic shock: clinical outcomes and impact of duration of hypotension prior to initiation of antimicrobial therapy. Intensive Care Med 35:871-881

22. Joannidis M, Metnitz B, Bauer P, Schusterschitz N, Moreno R, Druml W, Metnitz PG (2009) Acute kidney injury in critically ill patients classified by AKIN versus RIFLE using the SAPS 3 database. Intensive Care Med 35:1692-1702 